\title{
STATUS OF WOLF-RAYET MODELS
}

\author{
D.J. HILLIER \\ Department of Physics and Astronomy, University of Pittsburgh, 3941 O'Hara Street, \\ Pittsburgh, PA 15260, U.S.A.
}

\begin{abstract}
In the last decade there has been a tremendous advance in our understanding of the atmospheres and stellar winds of Wolf-Rayet (WR) stars. This has arisen through improved observations, and through the application of extensive radiative transfer codes which has allowed us to model some of the complex processes occurring in WR envelopes. Present models are able to simultaneously treat detailed atomic models for $\mathrm{H}, \mathrm{He}, \mathrm{C}, \mathrm{N}$, $\mathrm{O}$ and $\mathrm{Si}$. In the near future we will be able to treat $\mathrm{Fe}$, and to more accurately treat the formidable problem of non-LTE line-blanketing. In this review we address current limitations in modeling the extended atmospheres of WR atmospheres. These limitations fall into two classes - those reflecting our inability to accurately compute the "Standard Model", and those referring to assumptions in the "Standard Model" that need to be relaxed. Under the first category we include inaccuracies in the atomic data, the neglect of line-blanketing in the model calculations, and the inability of the present generation of models to compute the velocity law. Under the second category we include the assumption of spherical geometry, the assumption of homogeneity, the neglect of rotation, and the influence of $\mathrm{X}$-rays arising from shocks in the stellar wind.
\end{abstract}

Key words: stars: Wolf-Rayet - atmospheres - polarization - winds

\section{Introduction}

The accurate interpretation of WR spectra is extremely difficult because the observed spectrum is formed in a dense stellar wind in which the usual assumptions of plane parallel geometry and local thermodynamic equilibrium (LTE) are totally inadequate. At the very minimum one must adopt a spherical model and non-LTE. The latter is non-trivial, requiring the solution of the radiative transfer equation simultaneously with the statistical equilibrium equations for all important atomic species.

The present generation of models (Sect. 2.), while providing an excellent basis for the interpretation of WR spectra, fail to fully explain the observed spectra. The discrepancies are important since they provide a means of determining the reliability of the model assumptions, and the accuracy of the parameter determinations.

\section{The "Standard Model"}

The basic model generally adopted to analyze WR stars assumes that the observed emission line spectrum arises in a dense stellar wind which is photoionized by a hot core. With this basic premise several codes based on spherical geometry and non-LTE have been developed (Hamann 1985; Koesterke et al. 1992 [Kiel code]; Hillier 1990b) in which the line and contin- 
uum formation are treated simultaneously. Through a detailed comparison of continuum fluxes, line fluxes, and line profiles with those observed, stellar parameters can be derived.

The existing codes are based on several assumptions, and give rise to what is termed the "Standard Model" for WR stars. In addition to the basic premise previously alluded to, the "Standard Model" assumes spherical geometry, a monotonic velocity law, homogeneity, time independence, and statistical and radiative equilibrium. The assumption of radiative equilibrium has not always been included in model calculations (particularly in the wind) but it is included with the "Standard Model" assumptions since it is a simplifying assumption of similar quality. The "Standard Model" has been used with great success to analyse the spectra of WR stars. Reliable stellar parameters have been determined (Hamann et al. 1993; Crowther et al. $1994 \mathrm{a}, 1994 \mathrm{~d}$ and these proceedings), placing important constraints on the evolution of massive stars. The "Standard Model" has also provided an excellent basis for understanding line and continuum formation in dense stellar winds (e.g., Hillier 1991c). Through a systematic analysis of how lines form, we have identified those lines most suitable for abundance analysis (e.g., Hillier 1990a). Further, we have identified line ratios that are sensitive to the effect of line-blanketing, allowing a subtle test of the models when we include line blanketing into the calculations (Crowther et al. 1994a, 1994b).

Advances in modeling techniques (Rybicki 1991), expanded and improved observations, and advances in understanding wind dynamics (Lucy \& Abbott 1993; Springmann 1994; Gayley et al. 1994) now allow us to seriously consider both improving the computation of the "Standard Model", and relaxing some of its assumptions.

\section{Improvements to the "Standard Model"}

Many of the deficiencies in present model calculations of WR spectra (see, for example, spectral fits by Crowther et al. 1994a, 1994b), are not (necessarily) deficiencies in the "Standard Model", but are probably related to the following deficiencies in its computation:

a. inaccuracies in treating atomic process (due to data quality and neglect of important processes),

b. the neglect of non-LTE line-blanketing, and

c. the adoption of an ad hoc velocity law, rather than the determination of $v(r)$ self consistently using radiation hydrodynamics.

In general, models for WN stars are in much better agreement with observation than models for WC stars. This primarily reflects the difficulty of computing realistic spectra for WC stars since the atmospheres of these stars contain comparable amounts of $\mathrm{He}, \mathrm{C}$, and (perhaps) $\mathrm{O}$. 


\section{1 АTOMIC DATA}

In the last few years a tremendous amount of atomic data has become available through the opacity group (Cunto \& Mendoza 1992), and through calculations of atomic data for individual ions by other groups. While these data will allow considerable advances in model atmosphere calculations, it is important to remember that there are uncertainties associated with the atomic data which vary considerably from transition to transition. Even for species with "excellent" data, such as C III, certain transitions may have poorly known oscillator strengths (see, e.g., Hillier 1991c).

The inclusion of dielectronic recombination into model calculations is still a major difficulty. At present, low temperature dielectronic recombination (LTDR) is generally included using the method of Mihalas \& Hummer (1973) whereby the transitions are treated as lines. The recombination paths appear as resonances in the photoionization cross sections, and hence, in principle, can be treated directly. Unfortunately, there is still a tendency in many model calculations to ignore the resonances entirely.

Of more fundamental difficulty is the treatment of high temperature dielectronic recombination (HTDR). This recombination occurs to very high $n$ levels, is strongly density dependent, and is not allowed for in present WR models. To illustrate its possible importance, and using the rates tabulated by Nussbaumer \& Storey $(1983,1984)$, and by Aldrovandi \& Pequignot $(1973,1976)$, we note that for C III, HTDR exceeds, in the low density limit, both LTDR and radiative recombination at a temperature of only $20000 \mathrm{~K}$.

\subsection{NON-LTE LINE-BLANKETING}

Most models do not include the effects of non-LTE line-blanketing. Rapid progress is being made in this field, and considerable advances are expected in the next 5 years. The only non-LTE line-blanketing calculations for WR stars are by Schmutz (1990, 1994a, these proceedings). These calculations are based on the Kiel code, and a modified version of the Abbott-Lucy Monte-Carlo code. At present the calculations assume pure scattering, and treat the departures of the Fe levels from LTE in an approximate manner.

The influence of blanketing reveals itself in modeling through inconsistency of model fits. In particular, present models cannot simultaneously match the lowest and highest ionization stages. For example, in WNE stars the present generation of models cannot reproduce the $\mathrm{N} v$ spectrum and the $\mathrm{N}$ III spectrum simultaneously. This discrepancy is most readily explained as an effect of blanketing, and does not invalidate the "Standard Model".

A particularly striking example of the effects of line-blanketing is afforded from the study of Crowther et al. (1994a, 1994b) for WNL and Ofpe/WN stars. The effective temperature determination is based primarily on the ratio of He II $\lambda 4686$ to He I $\lambda 5876$. For HD 86161 (WR 16) excellent fits 
to the $\mathrm{H}$ and He spectrum are obtained for a model with $T_{\text {eff }}=26300 \mathrm{~K}$ and $\mathrm{R}(\tau=2 / 3)=26.7 \mathrm{R}_{\odot}$. In the IR region, however, the He I line at $2.058 \mu \mathrm{m}$ disagrees strongly with the observed profile. The model profile can be brought into agreement by lowering the effective temperature by only $1500 \mathrm{~K}$. The excellent fit to the other $\mathrm{He} \mathrm{I}$ and the $\mathrm{H}$ lines is maintained, but now He II $\lambda 4686$ is considerably weaker than observed.

The behaviour of HeI $2.058 \mu \mathrm{m}$ in Ofpe/WN9 stars is discussed in detail by Narrajo et al. (1994). It behaves differently from other He I lines because the upper level of the transition is also coupled to the ground state of neutral He by a transition at $584 \AA$. Only when the $584 \AA$ transition is optically thick, do we expect to see significant $2.058 \mu \mathrm{m}$ emission.

The difference in effective temperature between the models is comparable to what Schmutz (1991) estimated for the effect of line blanketing in detailed models of the Ofpe/WN9 star R84. In a similar vein it is found that the H/He model is unable to reproduce the $\mathrm{N}$ spectrum. To fit the $\mathrm{N}$ spectrum a model with a slightly higher effective temperature is required. The difference in stellar parameters must be regarded as indicative of current uncertainties in WR models.

Further evidence of the effects of line-blanketing is found from the analysis of WR nebulae by Esteban et al. (1993). They found that the pure $\mathrm{H} / \mathrm{He}$ models overestimate the extreme UV flux in WNL stars.

\subsection{Computation of the VElocity laW}

One of the major problems with the present analyses of WR stars is that the velocity law is assumed (or fitted by the observations) rather than found consistently with the radiation field (e.g., Hamann 1994). Typically a velocity law of the form $v(r)=\left(1-R_{*} / r\right)^{\beta}$ is assumed which is joined smoothly on to a hydrostatic structure at a velocity less than $100 \mathrm{~km} \mathrm{~s}^{-1}$. This velocity law, with $\beta \approx 1$, was adopted since it is found to be an excellent approximation to that found in $\mathrm{O}$ stars (observationally and theoretically).

Experimentation has shown that the radii of WR stars are not well determined by the present generation of models if $\beta$ is treated as a free parameter (e.g., Hillier 1991c) - higher $\beta$ values lead to smaller core radii. To be consistent with evolution calculations, which show that a pure He star of $10 \mathrm{M}_{\odot}$ has a radius of less than $1 \mathrm{R}_{\odot}$, it appears that a slow acceleration (large $\beta$ ) in the inner regions of the stellar wind is required. The spectral calculations are insensitive to the structure of this region because it is optically thick.

Clearly, if we wish to determine stellar radii, we need to derive the velocity law from first principles. Two approaches are possible:

a. We can deduce the velocity law from a modified CAK model.

b. We can determine the velocity law self-consistently using a full non-LTE line blanketed model.

Approach (a), advocated by Owocki, has considerable advantage in that it 
allows physical insight into the wind dynamics not readily available through detailed models. We recall that the CAK model made some very basic assumptions yet was able to reproduce the basic characteristics of $O$ star mass loss. Approach (b) is the optimal approach, but is also the most difficult. It is strongly related to the inclusion of non-LTE line blanketing in the "Standard Model". Once we have included non-LTE line-blanketing we can routinely compute the line force, and hence compute the velocity law, provided WR winds are indeed radiation driven.

Preliminary calculations by Springmann (1994) and Gayley et al. (1994) on line driving in WR stars suggest a slow velocity law (i.e., $\beta>1$ ), and that the simple $\beta$-velocity law is inappropriate.

\section{Relaxing assumptions in the "Standard Model"}

With the present generation of models, and their limitations, the "computational" evidence that the "Standard Model" is in conflict with real WR stars is, in general, sparse. At present there are two basic discrepancies between models and observation that might be attributed to deficiencies in the "Standard Model". These are:

(a) The present models for WNE stars produce P-Cygni absorption on He I 5876 and He I 10830 which is stronger than observed (e.g., Hamann 1994). Note that the comparison of the theoretical He I 5876 profile with that observed is not straight forward, since the blue side of the profile is blended with the CIV 5805 doublet, while the red side of the profile is contaminated by $\mathrm{NaD}$ and telluric absorption.

(b) The model electron scattering wings are stronger than observed (factor of 2?, Hillier 1991). Because of the difficulty in identifying the location of the true continuum, the detection and measurement of these wings is extremely difficult (particularly in WCE stars).

Many of the other discrepancies between theory and observation may be due to inadequacies of the "Standard Model" calculations (Sect. 2.). Other evidence for relaxing the "Standard Model" comes from observations:

1. Some Wolf-Rayet stars show intrinsic polarization. If the stars are single they cannot be spherically symmetric.

2. Many Wolf-Rayet stars are strong X-ray sources. X-rays cannot be generated in the "Standard Model".

3. Many Wolf-Rayet stars show profile variations, which are relatively small, and have been interpreted as inhomogeneities in the stellar wind.

Below we examine some facets of the "Standard Model" that may need to be relaxed in future models. Clumping will not be considered as it is discussed extensively elsewhere in this symposium. 


\subsection{Rotation}

In current spectral modeling of WR stars the effects of rotation on the observed spectrum are ignored. Rotational broadening effects in $\mathrm{O}$ stars are very large, and cannot be neglected. Many $O$ stars have rotation velocities of approximately 100 to $400 \mathrm{~km} \mathrm{~s}^{-1}$ which broaden all photospheric lines. Even for $\mathrm{H} \alpha$, rotational broadening is important in the core. Since there is a continuum of stars between the "pure" WR stars and "pure" O stars, rotation must also be important for the least extreme WR stars. Through the work of Bjorkman \& Cassinelli (1993), and Owocki et al. (1994) we now have a primitive understanding of how rotation can be incorporated into models of WR winds.

The effect of rotation on lines formed in the wind is generally considerably less important than for photospheric lines, because the rotational velocity is typically much less than the terminal velocity of the stellar wind. The only (published) work on the influence of rotation on wind profiles is for $\mathrm{O}$ stars by Mazzali (1990). Mazzali computed the effects of rotation on P-Cygni profiles as a function of rotation speed (relative to the terminal velocity). As expected, the influence on the line profiles increases as the rotational velocity increases.

Rotation considerably complicates the transfer of radiation since the velocity along a given sight line through the atmosphere is no longer monotonic. Thus the line transfer, even in the pure Sobolev case, is non local. For calculations in the comoving-frame the non monotonicity of the velocity law means that the usual practice of setting the boundary condition on the blue side of the line profile can no longer be adopted, considerably complicating the solution of the transfer equation.

For many WN stars the quality of fits to the H, He I, and He II profiles is excellent. The discrepancies are certainly at the level where rotation may be a key missing ingredient, and hence the effect of rotation must be included before we start using the discrepancies as diagnostics of other departures of WR atmospheres from the "Standard Model".

\subsection{Departures From SPhERICAL Geometry}

The assumption of "spherical geometry" in the "Standard Model" for WR stars obviously allows a great simplification, however, its adoption was not purely for convenience. Two lines of evidence suggest that the extended atmospheres of many WR stars are spherically symmetric.

a. In both WNE and WCE stars one, or more, profiles show flat tops. The EASIEST way to produce such a profile is through emission from an optically thin, spherically symmetric, hollow shell. In WNE and WCE stars the lines that are observed to be flat topped are the lines that the models predict to be flat topped. 
b. There is an apparent absence of double peaked profiles, as seen, for example in Be stars and T Tauri stars. Profiles in these stars tend to emphasize that the circumstellar material is not spherically distributed.

The problem with both pieces of evidence is that we have not yet determined what constraints this places on the departures from spherical geometry. Is the maximum variation in the wind density (at a given radius) only $10 \%$, or could there be considerably larger departures (factors of 2,10 ) if we take into account observational errors, effects of line blending, and inclination effects, etc.? Evidence has recently come forward that some WR stars are intrinsically polarized, indicating significant departures from spherical symmetry. In addition, two luminous blue variables (LBVs) are known to show intrinsic polarization and, further, many LBVs reside in ejected nebulae which are non-spherical. For AG Car, the (almost) symmetric electron scattering wings on $\mathrm{H} \alpha$ are seen to have enhanced polarization in one data set, as expected for an asymmetric wind (Schulte-Ladbeck et al. 1994). The deduced position angle is parallel to the major axis of the nebulae.

Work on interpreting polarization observations of stars with dense extended stellar winds is still in its infancy. A fundamental result was derived by Brown and McLean (1977) who found that the polarization of an optically thin axisymmetric envelope could be written in the form $P=$ $0.375 \bar{\tau}(1-3 \gamma) \sin ^{2} i$ where $\bar{\tau}$ represents the angle averaged optical depth of the envelope, $\gamma$ is the shape factor describing the shape of the envelope, and $i$ is the inclination of the observer relative to the symmetry axis. This simple result shows the difficulty in interpreting polarization observations of single stars - from a single polarimetric observation it is impossible to determine the shape of the envelope since its optical depth, and the viewing inclination, are just as important in determining the observed polarization.

For WR stars the simple result must be regarded as illustrative only, since multiple scattering, and absorptive opacity, can strongly modify the observed polarization. The importance of multiple scattering effects, and the influence of absorptive opacity, were highlighted in a recent paper by Hillier (1994a). For sufficiently large optical depths, multiple scattering can cause the polarization to reverse sign (equivalent to a change of $90^{\circ}$ in the direction of the net electric vector in the plane of the sky).

Work on the calculation of polarized line profiles due to electron scattering is even more limited. Wood, Brown \& Fox (1993) use analytical techniques to illustrate some of the effects that might be expected to be seen by polarimetric line observations. Hillier (in preparation) has just begun to compute polarized line profiles for realistic model atmospheres using 2 distinct approaches - a Monte-Carlo method, and a second method which solves the transfer equation for the transfer of polarized radiation explicitly. These calculations indicate the importance of allowing for line absorption, stratification, and for frequency redistribution effects arising when photons 
are scattered by electrons in motion.

Considerable advances in the interpretation of polarization observations of WR stars (and LBVs) should be made in the next few years. In order to distinguish between various competing effects it will be necessary to simultaneously model the I, Q and U Stokes parameters for both lines and the continuum.

\subsection{X-RAYS}

One of the outstanding results of the Einstein satellite is that both $\mathrm{O}$ and WR stars are strong X-ray sources (Seward et al. 1979; Pollock 1987). More recently, high quality ROSAT observations have become available (Wessolowski et al. these proceedings) allowing improved determinations of the observed X-ray luminosities. For single stars the observed X-rays probably originate from shocks in the stellar wind. These shocks are expected to arise from the line driven wind instability (e.g., Owocki et al. 1988). The only detailed work on the influence of X-rays on the emergent spectrum of WR stars is by Baum et al. (1992), who considered the WNE-w star HD 193077 (WR138). Baum et al. assumed that X-rays arose through free-free emission in a stellar wind. The filling factor and shock temperature were treated as free parameters, and were chosen to match the observed Einstein X-ray luminosity. Two important conclusions can be drawn from this work:

a. The inclusion of X-rays did not seriously modify the stellar parameters found from spectral fitting of the $\mathrm{H}$ and He lines. Some enhancement in the strength of line from high ionization species was seen $(e . g ., \mathrm{N} \mathrm{v})$ which may help to alleviate some of the difficulties in simultaneously fitting all ionization stages of $\mathrm{N}$ simultaneously.

b. The abundance of high ionization (impurity) species was considerably enhanced in the outer regions of the stellar wind. This enhancement arose through the Auger process in which 2 electrons are ejected.

Due to the importance of $\mathrm{X}$-rays in fine tuning WR models, theoretical guidance on X-ray production in WR winds is needed. In particular, how does the $\mathrm{X}$-ray emission vary with radius? Unfortunately there is a large degree of freedom with the inclusion of X-rays in model calculations since the constraints supplied by existing $\mathrm{X}$-ray observation are rather limited, as revealed by the following considerations:

a. Interstellar absorption is strong, particularly at soft $(<1 \mathrm{keV}) \mathrm{X}$-ray energies. Unfortunately most WR stars are heavily reddened. For example, the average $E_{B}-v$ of the Crowther sample of WN7-WN8 stars is 0.64, corresponding to a $\mathrm{H}$ column density of $3.7 \times 10^{21} \mathrm{~cm}^{-2}$ and an optical depth of approximately 1 at $1 \mathrm{keV}$ (Shull \& Van Steenburg 1985).

b. The optical depth of a WR envelope is very large. Consequently, the observed X-rays (particularly at energies $<1 \mathrm{keV}$ ) can only originate in the outer regions (radio emitting region) of the stellar wind. Unfortu- 
nately, X-ray emission from large radii is of no great importance, as far as spectral modeling is concerned, since it is unlikely that $\mathrm{X}$-rays generated at a few $R_{*}$ will be characterized by the same filling factor and shock temperature as those formed at $1000 R_{*}$.

Pauldrach et al. (1994) note that shocks also modify the soft UV radiation field, and that this, as well as Auger ionization, can significantly modify the ionization structure.

Recent ROSAT observations of $\zeta$ Pup (Hillier et al. 1993) provide an excellent example of how the wind can modify the observed X-ray spectrum. The observed X-ray spectrum of $\zeta$ Pup is best fitted by a model in which $\mathrm{He}^{2+}$ recombines to $\mathrm{He}^{+}$in the outer region of the stellar wind, as predicted by recent spectral modeling of $\zeta$ Pup (Pauldrach et al. 1994). Models'in which He remains doubly ionized show a significant $\mathrm{K}$ shell absorption which is much stronger than that observed.

\section{Conclusions}

Considerable advances in the modeling of WR spectra has occurred over the last decade. These advances have allowed the derivation of fundamental stellar parameters providing invaluable constraints on evolutionary calculations. In addition, the models have provided insights into line and continuum formation in the extended atmospheres of WR stars. With recent improvements in radiative transfer techniques, and the availability of the necessary atomic data, we are now in a position to considerably improve our models. The next generation of models will address many of the problems with current spectral fits, improve our understanding of physical processes in WR winds, and provide even tighter constraints on the evolution of massive stars.

\section{Acknowledgements}

The author gratefully acknowledges partial financial support from Space Telescope (GO-4450.01-92A, GO-5460.01-93A).

\section{References}

Aldrovandi, S.M.V., Pequignot, D. 1973, $A \& A$ 25, 137

Aldrovandi, S.M.V., Pequignot, D. 1976, $A \mathscr{E} A$ 47, 321

Baum, E., Hamann, W.-R., Koesterke, L., Wessolowski, U. 1992, A\&A 266, 402

Bjorkman, J.E., Cassinelli, J.P. 1993, ApJ 409, 429

Brown, J.C., McLean, I.S. 1977, $A \& A$ 57, 141

Crowther, P.A, Hillier, D.J., Smith, L.J. 1994a, $A \mathscr{B} A$ in press

Crowther P.A, Hillier, D.J., Smith, L.J. 1994b, $A \mathscr{E} A$ in press

Crowther, P.A, Smith, L.J., Hillier, D.J., Schmutz, W. 1994d, $A \& A$ in press

Cunto, W., Mendoza, C. 1992, Rev. Mexicana Astron. Astrophys. 23, 107

Esteban, C., Smith, L.J., Vilchez, J.M., Clegg, R.E.S. 1993, $A \& A$ 272, 299 
Gayley, K.G., Owocki, S.P., Cranmer, S.R. 1994, ApJ submitted

Hamann, W.R. 1985, $A \mathcal{E} A$ 148, 364

Hamann, W.-R. 1994, in: D. Vanbeveren, W. van Rensbergen \& C. de Loore (eds.), Evolution of Massive Stars: A Confrontation between Theory and Observation (Dordrecht: Kluwer), p. 237

Hamann, W.-R., Koesterke, L., Wessolowski, U. 1993, $A \cup A$ 274, 397

Hillier, D.J. 1990a, in: C. Garmany (ed.), Intrinsic Properties of Hot Luminous Stars, ASP Conf. Series 7, p. 340

Hillier, D.J. 1990b, $A \mathscr{E A}$ 231, 116

Hillier, D.J. 1991a, $A \& A$ 247, 455

Hillièr, D.J. 1991 b in: L. Crivellari, I. Hubeny \& D.G. Hummer (eds.), Stellar Atmospheres: Beyond Classical Models, NATO ASI Series C941 (Kluwer: Dordrecht), p. 317

Hillier, D. J. 1994a, $A \mathscr{B} A$ (in press)

Hillier, D.J., Kudritzki, R.P., Pauldrach, A.W., Baade, D., Cassinelli, J.P., Puls, J., Schmitt, J.H.M.M. 1993, $A \mathscr{B} A$ 276, 117

Koesterke L., Hamann, W.-R., Kosmol, P. 1992, $A \& A$ 255, 490

Lucy L.B., Abbott, D.C. 1993, ApJ 405, 738

Mazzali, P.A. 1990, $A \& A$ 238, 191

Mihalas, D., Hummer, D.G. 1973, ApJ 179, 827

Najarro, F., Hillier, D.J., Kudritzki, R.P., Krabbe, A., Genzel, R., Lutz, D., Drapatz, D., Geballe, T.R. 1994, $A \& A$ 285, 573

Nussbaumer, H., Storey, P.J. 1983, $A \mathcal{E} A$ 126, 75

Nussbaumer, H., Storey, P.J. 1984, A\&A Suppl. 56, 293

Owocki, S.P., Castor, J.I., Rybicki, G.B. 1988, $A p J$ 335, 914

Owocki, S.P., Cranmer, S.R., Blondin, J.M. 1994, $A p J$ 424, 887

Pauldrach, A.W.A., Kudritzki, R.P., Puls, J., Butler, K., Hunsiger, J. 1994, $A \& A$ 283, 525

Pollock, A.M.T. 1987, ApJ 320, 283

Rybicki, G.B. 1991 in: L. Crivellari, I. Hubeny \& D.G. Hummer (eds.), Stellar Atmospheres: Beyond Classical Models, NATO ASI Series C341 (Kluwer: Dordrecht), p. 1

Schmutz, W. 1990, in: C. Garmany (ed.), Intrinsic Properties of Hot Luminous Stars, ASP Conf. Series 7, p. 117

Schmutz, W. 1991, in: L. Crivellari, I. Hubeny \& D.G. Hummer (eds.), Stellar Atmospheres: Beyond Classical Models, NATO ASI Series C341 (Kluwer: Dordrecht), p. 191

Schmutz, W. 1994a, in: D. Vanbeveren, W. van Rensbergen \& C. de Loore (eds.), Evolution of Massive Stars: A Confrontation between Theory and Observation (Dordrecht: Kluwer), p. 253

Schulte-Ladbeck, Clayton, G.C., Hillier, D.J., Harries, T.J., Howarth, I.D. 1994, ApJ in press (July)

Schulte-Ladbeck, R.E., Meade, M.R., Hillier, D.J., 1992 in: L. Drissen, C. Leitherer \& A. Nota (eds.), Nonisotropic and Variable Outflows from Stars, ASP Conf. Series 22, p. 118

Seward, F.D., Forman, W.R., Giacconi R., Griffiths, R.E., Harnden, F.R., Jones, C., Pye, J.P. 1979, ApJ (Letters) 234, L55

Springmann, U. 1994, $A \mathcal{E} A$ in press

Shull, J.M., Van Steenberg, M.E. 1985, $A p J$ 294, 599

Wood, K., Brown, J.C., Fox, G.K. 1993, $A \& A$ 271, 492 


\section{DISCUSSION:}

Lindsey Smith: I think the wings are probably present. We saw them clearly in some of the Atlas stars and Castor modeled them (ApJ Letter 1969). I agree with you that probably we cut them off.

Hillier: I would concur that the wings are present, however, I think that considerably more observational work needs to be done on their detection and measurement, since they are an important diagnostic tool of the wind structure.

Koenigsberger: If the wind is permeated by shocks, what does this do to the assumption of radiative equilibrium?

Hillier: I think the best answer to this question comes from a comparison of our non-LTE models with the observed spectra of WR stars. In particular the comparison of the equivalent width of IR HeII lines with models, and the modelling of UV collisional lines (and optical recombination lines) suggest that the assumption of radiative equilibrium is reasonable.

Conti: I'd like to know more about the typical asymmetry in W-R stars. Is 2:1 typical, a maximum, or a minimum?

Hillier: Unfortunately I am not yet in a position to specify the maximum density contrast that is still consistent with WR observations. The factor of 2 to $1 \mathrm{I}$ used in the model comes from the requirement that the model produce a polarization of approximately $0.5 \%$. Such a level of polarization is observed in $\mathrm{HD} 50896$, and some other W-R stars, but it is still uhncertain what this implies for the general asymmetry of W-R winds.

Cherepashchuk: Did you try to calculate the eclipsing effects in polarisation for WR $+\mathrm{O}$ binaries. Recently St-Louis, Moffat and collaborators carried out interpretation of polarimetric curve of V444 Cyg during secondary eclipse in the framework of a spherical model of WN5 star wind. Could you calculate the theoretical eclipsing polarisation curves in the model of a nonspherical wind?

Hillier: It is possible to modify the code and compute the polarization light curve for a binary system. My main concern in performing this calculation regards the influence of the Bow shock on the polarization light curve. Perhaps simple analytical models, or even 2D \& 3D simulations of colliding winds, could be used to provide a guide as to the importance of the Bow shock. 